ISSN 0103-9954

\title{
INFLUÊNCIA DE PROTETORES FÍSICOS COLORIDOS NAS TROCAS GASOSAS EM MUDAS DE CANAFÍSTULA [Peltophorum dubium (Spreng.) Taub.]
}

\author{
INFLUENCE OF COLORED PHYSICAL PROTECTORS ON GAS EXCHANGES OF \\ CANAFISTULA [Peltophorum dubium (Spreng.) Taub.] SEEDLINGS
}

\author{
Jeferson Klein ${ }^{1}$ Leandro Rampim ${ }^{2}$ Débora Kestring ${ }^{3}$ Vandeir Francisco Guimarães ${ }^{4}$ \\ João Domingos Rodrigues ${ }^{5}$
}

\begin{abstract}
RESUMO
A utilização de protetores físicos é uma técnica eficiente na semeadura direta de espécies nativas. Devido à importância da espécie Peltophorum dubium para a utilização no repovoamento de áreas degradadas, arborização e paisagismo, determinou-se o comportamento fisiológico das plantas com a análise da influência de um protetor físico com diferentes taxas de luminosidade no processo das trocas gasosas das plantas. O experimento foi conduzido em vasos com o solo Latossolo Vermelho Distroférrico, típico da região, em vasos com solo. As plantas de Peltophorum dubium foram avaliadas aos 30, 50, 70, 90, 110 e 130 dias após a semeadura (DAS) com os tratamentos: T1, ausência de protetor físico (APF); T2, protetor físico transparente (PFT); T3, protetor físico transparente + celofane azul (PFA) e T4, protetor físico transparente + celofane vermelho (PFV). Foram avaliadas as seguintes características: condutância estomática, transpiração, assimilação de $\mathrm{CO}_{2}$, concentração de $\mathrm{CO}_{2}$ no interior da câmara subestomática e eficiência do uso de água. Os resultados obtidos mostraram que o protetor físico independentemente de sua coloração promoveu aumento dos valores médios de trocas gasosas durante a permanência dos protetores físicos no ponto de semeadura 70 DAS, exceto para assimilação líquida de $\mathrm{CO}_{2}$. No entanto, a eficiência do uso de água foi maior com APF 70 DAS.
\end{abstract}

Palavras-chave: condutância estomática; transpiração; assimilação de $\mathrm{CO}_{2}$; eficiência do uso de água.

\begin{abstract}
The utilization of physical protectors has been considered an efficient technique for direct sowing of different species, mainly native ones. Based on the importance of the species Peltophorum dubium for revegetation of degraded areas, tree planting and landscaping, this study evaluated gas exchanges in canafistula seedlings under the influence of physical protectors subjected to different luminosity rates. The experiment was carried out in pots a Dystropheric Red Latosol, a typical soil of the study region. Seedlings of Peltophorum dubium were evaluated at 30, 50, 70, 90, 110, and 130 days after sowing (DAS). Thus, the following treatments were adopted: T1, absence of physical protector (APP); T2, transparent physical protector (TPP); T3, transparent physical protector + blue cellophane (BPP) and T4, transparent physical
\end{abstract}

1 Biológo, Dr., Professor da Escola Politécnica, Pontifícia Universidade Católica do Paraná, Av. da União, 500, Jardim Coopagro, CEP 85902-532, Toledo (PR), Brasil. jeferson.klein@pucpr.br

2 Engenheiro Agrônomo, Dr., Professor da Universidade Estadual do Centro Oeste-UNICENTRO, campus Cedeteg, Setor de Ciências Agrárias e Ambientais, Departamento de Agronomia, Rua Simeão Varela de Sá, 03, Vila Carli, CEP 85040-080, Guarapuava (PR), Brasil. rampimleandro@yahoo.com.br

3 Biológa, Dra ${ }^{\text {. }}$, Pesquisadora do Programa Nacional de Pós-Doutorado da Universidade Estadual do Oeste do Paraná, Rua Pernanbuco, 1777, Caixa Postal 91, Centro, CEP 85960-000, Marechal Cândido Rondon (PR), Brasil. deborakestring@yahoo.com.br

4 Engenheiro Agrônomo, Dr., Professor da Universidade Estadual do Oeste do Paraná, Rua Pernanbuco, 1777, Caixa Postal 91, Centro, CEP: 85960-000, Marechal Cândido Rondon (PR), Brasil. vandeirfg@yahoo.com.br

5 Engenheiro Agrônomo, Dr., Professor do Instituto de Biociências de Botucatu, Universidade Estadual de São Paulo, Caixa Postal 510, Distrito de Rubião Júnior, CEP: 18618-970, Botucatu (SP), Brasil. mingo@ibb.unesp.br

Recebido para publicação em 27/10/2011 e aceito em 17/10/2014

Ci. Fl., v. 26, n. 3, jul.-set., 2016 
protector + red cellophane (RPP). The evaluated characteristics were: stomatal conductance, transpiration, $\mathrm{CO}_{2}$ assimilation, $\mathrm{CO}_{2}$ concentration inside the substomatal chamber and water use efficiency. In general, all types of physical protectors led to higher mean values of gas exchanges during their permanence in the sowing site until 70 DAS, except for $\mathrm{CO}_{2}$ assimilation. However, water use efficiency was higher in APP seedlings at the same period.

Keywords: Stomatal conductance; transpiration; $\mathrm{CO}_{2}$ assimilation; water use efficiency.

\section{INTRODUÇÃO}

As formações florestais do Brasil vêm sofrendo níveis significativos de degradação, devido ao avanço da fronteira agropecuária, construção de usinas hidrelétricas (LIMA-JUNIOR et al., 2005; MALAVASI et al., 2010; STÜMER et al., 2011), e ocupação humana (AVILA et al., 2011). Conforme Hansen et al. (2008), o Brasil foi responsável por $47,8 \%$ do desmatamento mundial de florestas tropicais úmidas entre 2000 e 2005 , quatro vezes maior que a Indonésia, segunda colocada, com $12,8 \%$. Tal levantamento revela o atual cenário mundial, em que o desmatamento acumulado neste período foi de $2,72 \times 10^{5} \mathrm{~km}^{2}$, o equivalente a uma área maior que a dos estados de São Paulo e Sergipe juntos e somente a taxa anual de desmatamento no território nacional foi de $26 \mathrm{mil} \mathrm{km}^{2}$. Na última avaliação realizada pelo Instituto Nacional de Pesquisas Espaciais (INPE), o estado de São Paulo apresentou aumento de nove vezes no nível de desmatamento da Mata Atlântica desde 2005 (INPE, 2009). Contudo, a necessidade da recomposição florestal demanda informações sobre a forma correta, eficiente e de baixo custo para se efetuar a introdução de espécies arbóreas nas áreas em que houve retirada da floresta nativa, assim como em áreas com solo contaminado (SILVA et al., 2011).

A canafístula ou faveiro [Peltophorum dubium (Spreng.) Taub.] é uma espécie nativa frequente em todo o domínio da floresta estacional semidecidual, bem como no cerrado e abundante em formações secundárias (DONADIO e DEMATTÊ, 2000). No contexto ecológico, a espécie é classificada como pioneira oportunista, tolerante às altas taxas de luminosidade e temperatura $\mathrm{e}$ é considerada de grande potencial para uso em plantios mistos destinados à recomposição de áreas degradadas e preservação permanente (LORENZI, 2000).

Desta forma, a semeadura direta tem sido uma técnica versátil no processo de reflorestamento (BARNETT e BAKER, 1991). Segundo Ferreira et al. (2007), consiste na dispersão de espécies florestais cujas sementes são espalhadas diretamente no local a ser reflorestado. Tal técnica tem vantagem na redução do custo de implantação de povoamentos florestais, tendo em vista a eliminação da fase de viveiro e de mão de obra, ampliando sua importância em áreas carentes de recursos ou de difícil acesso (SOARES e RODRIGUES, 2008). Porém, Derr e Mann-Junior (1971) realizaram diversos trabalhos com semeadura direta e observaram que uma única técnica não foi suficiente para proteger as plantas contra as condições adversas.

Nesse sentido, o uso de protetores físicos junto à semeadura direta, torna-se relevante, uma vez que reduz a predação de sementes e plantas, além de poder interferir diretamente na geminação e estabelecimento das espécies por criar um microambiente favorável (FERREIRA et al., 2002; MATTEI e ROSENTHAL, 2002). Assim, diferentes materiais de vários tamanhos, formatos e cores, têm sido utilizados como protetores físicos ao longo do tempo em diferentes países (BARNETT e BAKER, 1991; SERPA e MATTEI, 1999; MATTEI et al., 2001; FERREIRA et al., 2007; BRACHTVOGEL et al., 2008; KLEIN et al., 2012).

As determinações de variáveis ecofisiológicas possibilitam a compreensão do comportamento vegetativo das plantas, assim como o seu desempenho pontual em relação às respostas aos tratamentos impostos (NOGUEIRA e SILVA-JUNIOR, 2001). Desta forma, a quantificação das trocas gasosas realizadas nas folhas, compreendendo assimilação líquida de $\mathrm{CO}_{2}$, transpiração, condutância estomática, concentração interna de $\mathrm{CO}_{2}$ na câmara subestomática, eficiência do uso de água, entre outros, estão intimamente relacionada ao estado hídrico do vegetal, além de seu desenvolvimento (NOGUEIRA et al., 2000). Da mesma forma, a qualidade e a intensidade da luz são fatores ambientais que podem influenciar nas trocas gasosas ocorridas nas plantas (COSTA e MARENCO, 2007).

Dentre os fatores físicos do ambiente, a luz desempenha papel relevante na regulação da produção primária, contribuindo de forma efetiva 
para o crescimento das plantas (DOUSSEAU et al., 2007). Porém, a resposta ou a sensibilidade das sementes à luz é específica para cada espécie (FERRAZ-GRANDE e TAKAKI, 2006). É válido mencionar que as respostas morfofisiológicas dos vegetais não dependem apenas da presença, atenuação ou ausência da luz, mas também da qualidade espectral (MARTINS et al., 2008). Nesse sentido, a faixa compreendida entre 400 e $480 \mathrm{~nm}$ representa a luz azul, que possui importante papel físiológico no desenvolvimento das plantas em aspectos como abertura estomática, alongamento do caule e direcionamento do crescimento (TAIZ e ZEIGER, 2013). Segundo Kinoshita et al. (2001), a adição de luz azul leva ao aumento considerável na abertura estomática. Conforme Srivastava e Zeiger (1995), a abertura estomática segue a radiação fotossinteticamente ativa na superfície da folha, em que a maior abertura estomática está diretamente relacionada à maior taxa de luminosidade (TAIZ e ZEIGER, 2013).

Tendo em vista todos os aspectos abordados, este trabalho objetivou avaliar a influência de protetores físicos coloridos nas trocas gasosas em mudas de canafístula [Peltophorum dubium (Spreng.) Taub.] de diferente idades.

\section{MATERIAL E MÉTODOS}

O presente ensaio foi conduzido em área aberta ( $22^{\circ} 52^{\prime}$ 'de latitude, $48^{\circ} 26^{\prime}$ de longitude e 822 $\mathrm{m}$ de altitude), do Departamento de Botânica do Instituto de Biociências, da Universidade Estadual Paulista "Júlio de Mesquita Filho" (Unesp), em Botucatu - SP, no período de agosto a dezembro de 2007. O local apresenta temperatura média anual de $19^{\circ} \mathrm{C}$ e umidade relativa do ar ao redor de $58 \%$ (AGRITEMPO, 2011). Já, os dados de temperatura do ar e umidade relativa do ar do período experimental foram obtidos na estação experimental da Faculdade de Ciências Agronômicas, Unesp, Botucatu - SP.

O solo utilizado no experimento apresentou as seguintes características químicas: $\mathrm{pH} \mathrm{CaCl}_{2}=$ 5,61; M.O. $=22,66 \mathrm{~g} \mathrm{~kg}^{-1} ; \mathrm{P}=22,8 \mathrm{mg} \mathrm{dm}^{-3} ; \mathrm{H}+\mathrm{Al}=$ $34,1 \mathrm{mmol}_{\mathrm{c}} \mathrm{dm}^{-3} ; \mathrm{K}=5,12 \mathrm{mmol}_{\mathrm{c}} \mathrm{dm}^{-3} ; \mathrm{Ca}=43,56$ $\mathrm{mmol}_{\mathrm{c}} \mathrm{dm}^{-3} ; \mathrm{Mg}=17,65 \mathrm{mmol}_{\mathrm{c}} \mathrm{dm}^{-3} ; \mathrm{SB}=66,3$ $\mathrm{mmol}_{\mathrm{c}} \mathrm{dm}^{-3} ; \mathrm{CTC}=100,4 \mathrm{mmol}_{\mathrm{c}} \mathrm{dm}^{-3}$ e V\% $=66$ $\%$. O solo é classificado como Latossolo Vermelho Distroférrico (EMBRAPA, 2013), característico da região.

Inicialmente, frutos maduros de canafístula
(Peltophorum dubium) foram coletados em diversas matrizes no mês de setembro de 2007, no Campus da Unesp de Botucatu - SP. Após a coleta, os frutos foram levados ao Laboratório de Relações Hídricas do Departamento de Botânica, no Instituto de Biociências da Unesp, Botucatu - SP, onde foram abertos para a remoção das sementes, excluindose aquelas que, aparentemente, encontravam-se danificadas por patógenos e predadores, bem como as de tamanho reduzido ou malformadas. Em seguida, as sementes foram submetidas à superação de dormência através de escarificação mecânica manual com lixa P 80. Posteriormente, foram imersas e mantidas em água à temperatura ambiente, por 12 horas, de modo que foram selecionadas para a semeadura apenas aquelas que apresentaram dormência superada, comprovada por sinais de embebição.

A semeadura foi manual e realizada a uma profundidade de aproximadamente $0,5 \mathrm{~cm} \mathrm{em}$ vasos de polietileno preto de $12 \mathrm{~L}$. O delineamento experimental utilizado foi blocos casualizados, em esquema fatorial $4 \times 6$ (tratamentos x épocas de coleta) com cinco repetições, totalizando 120 unidades amostrais.

O protetor físico utilizado constituiu-se de garrafas plásticas tipo polietileno tereftalato (P.E.T.), com volume de $2500 \mathrm{~mL}$ sem fundo e tampa, medindo $28 \mathrm{~cm}$ de altura e $36 \mathrm{~cm} \mathrm{de}$ diâmetro. As garrafas foram fixadas com varetas de bambu e fitas adesivas transparentes. Em alguns tratamentos, as garrafas foram recobertas com dupla folha de papel celofane nas colorações azul e vermelho. Assim, os tratamentos utilizados foram: T1 - ausência de protetor físico (APF); T2- protetor físico transparente (PFT); T3- protetor físico + celofane azul (PFA) e T4- protetor físico + celofane vermelho (PFV).

As avaliações de trocas gasosas foram realizadas periodicamente em seis momentos, dos 30 aos 130 dias após a semeadura (DAS), com intervalos de 20 dias. Durante as três primeiras avaliações (dos 30 aos 70 DAS), os protetores físicos, quando presentes, foram retirados momentos antes da determinação das trocas gasosas. Após 70 DAS, todos os protetores físicos foram retirados definitivamente.

As determinações das trocas gasosas foram realizadas utilizando-se equipamento de sistema fechado portátil de fotossíntese, Infrared Gas Analyzer (IRGA), modelo LI-6400, da LiCor, Lincoln, NE, EUA, sempre na região mediana 
do segundo par de folhas novas, completamente expandidas e totalmente expostas à radiação solar. Determinações das taxas de assimilação líquida de $\mathrm{CO}_{2}\left(A, \mu \mathrm{mol} \mathrm{CO} \mathrm{C}^{-2} \mathrm{~s}^{-1}\right)$, condutância estomática $\left(g_{s}, \mathrm{mmol} \mathrm{H}_{2} \mathrm{O} \quad \mathrm{m}^{-2} \mathrm{~s}^{-1}\right)$, transpiração ( $E, \mathrm{mmol}$

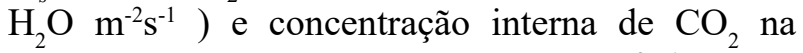
câmara subestomática $\left(\mathrm{Ci}, \mu \mathrm{mol} \mathrm{CO} \mathrm{CO}^{-2} \mathrm{~s}^{-1}\right)$ foram realizadas no período compreendido entre 8 e $11 \mathrm{~h}$. Também foi determinada a eficiência do uso de água $\left(E U A, \mathrm{~mol} \mathrm{~m}^{-2} \mathrm{~s}^{-1}\right)$, que correspondeu à relação entre a quantidade de $\mathrm{CO}_{2}$ assimilado por unidade de água perdida pela transpiração. Outra variável avaliada foi a taxa de fótons fotossinteticamente ativos no interior do ambiente. No experimento foi utilizado o nível de radiação de $1500 \mu \mathrm{mol}$ de fótons $\mathrm{m}^{-2} \mathrm{~s}^{-1}$.

Os resultados obtidos foram submetidos à análise de variância utilizando-se o programa computacional SISVAR (FERREIRA, 2010). Para analisar as trocas gasosas em cada época de avaliação em função dos protetores físicos foi aplicado Teste de Tukey e para observar o comportamento dos protetores físicos em função das épocas foi empregado a análise de regressão, conforme Banzatto e Kronka (1989). Posteriormente, modelos de regressão polinomial foram ajustadas com o programa SIGMA PLOT (Systat Software, Inc., 2011).

\section{RESULTADOS E DISCUSSÃO}

\section{Condutância estomática}

Foi detectada interação significativa entre protetores físicos e épocas de avaliação para a condutância estomática, em nível de $1 \%$ de probabilidade de erro. Nas avaliações realizadas até os 70 DAS, os protetores físicos, independentemente de sua coloração, promoveram aumentos significativos na condutância estomática das folhas (Tabela 1). Em café (Coffea arabica L.), as maiores taxas de condutância estomática para o cultivar Rubi foram observadas nas plantas cultivadas a $70 \%$ de sombreamento quando comparada à condição de pleno sol (FREITAS et al., 2003).

No presente estudo, as plantas submetidas ao tratamento com protetor físico transparente apresentaram redução gradual na condutância estomática ao longo das três primeiras avaliações. Em contrapartida, as plantas submetidas ao tratamento com protetor físico vermelho apresentaram aumento na condutância estomática ao longo do mesmo período, de modo que o maior valor médio foi observado aos 70 DAS. Dentre todas as plantas submetidas a protetores físicos, as folhas daquelas mantidas sob protetor físico azul apresentaram as menores variações nesta variável até 70 DAS. Além disso, até 70 DAS, os menores valores médios de condutância estomática foram detectados nas plantas que não foram submetidas a protetores físicos (APF).

O valor médio da condutância estomática obtido aos 30 DAS em folhas de canafístula cultivadas na ausência de protetores físicos apresentou valores percentuais inferior a 98,93 e 92 $\%$, comparados àqueles obtidos pelos tratamentos com protetores físicos transparente, azul e vermelho respectivamente.

Apesar da redução na condutância estomática das folhas de canafístula ter sido observada aos 90 DAS nas plantas submetidas a tratamentos com protetores físicos, não houve diferença significativa (Figura 1).

Ramos e Grace (1990) avaliaram a condutância estomática de folhas de algumas espécies pioneiras tolerantes e sensíveis à sombra e observaram que os maiores valores para esta característica foram obtidos em plantas cultivadas a pleno sol. Da mesma forma, Lima-Júnior (2005) constataram que plantas de Cupania vernalis Camb. apresentaram maior plasticidade em relação aos diferentes níveis de irradiação avaliados, uma vez que foram detectadas alterações anatômicas nas folhas de tais plantas, o que interferiu nas trocas gasosas, principalmente na condutância estomática.

No presente trabalho, provavelmente $o$ microambiente criado dentro dos protetores físicos, independentemente de sua coloração, por apresentar temperatura e umidade superiores, promoveu alterações no desenvolvimento inicial das plantas de canafístula nas condições testadas, tendo em vista que tais plantas apresentavam aumento da expansão da área foliar e limbos mais finos em comparação às folhas das plantas crescidas na ausência de protetores físicos.

Aos 110 DAS, as temperaturas foram superiores a $33^{\circ} \mathrm{C}$ e a umidade relativa do ar, inferior a $31 \%$, foram observados os menores valores médios de condutância estomática em todas as plantas, independentemente do tratamento. A redução no balanço entre entrada e saída de vapor d'água e $\mathrm{CO}_{2}$ pelas células-guarda era esperado, pois reflete o mecanismo adaptativo normal e específico das plantas, em função do deficit hídrico temporário. Este processo faz com que os estômatos se fechem 
TABELA 1: Valores médios da condutância estomática $\left(g_{s}\right.$, mmol $\left.\mathrm{H}_{2} \mathrm{O} \mathrm{m}^{-2} \mathrm{~s}^{-1}\right)$, de folhas de plantas de canafístula [Peltophorum dubium (Spreng.) Taub.], submetidas a diferentes qualidades de luminosidade.

TABLE 1: Mean values of stomatal conductance $\left(g_{s}, \mathrm{mmol} \mathrm{H}_{2} \mathrm{O} \mathrm{m}^{-2} \mathrm{~s}^{-1}\right)$, leaves of seedlings of canafístula [Peltophorum dubium (Spreng.) Taub.], subjected to different qualities of light.

\begin{tabular}{ccccccc}
\hline \multirow{2}{*}{ Tratamento } & \multicolumn{7}{c}{ Dias após a semeadura (DAS) } \\
\cline { 2 - 7 } & 30 & 50 & 70 & 90 & 110 & 130 \\
\hline APF & $0,12 \mathrm{c}$ & $0,39 \mathrm{c}$ & $0,36 \mathrm{c}$ & $0,93 \mathrm{a}$ & $0,06 \mathrm{a}$ & $0,39 \mathrm{a}$ \\
PFT & $3,95 \mathrm{a}$ & $2,21 \mathrm{a}$ & $1,25 \mathrm{~b}$ & $1,24 \mathrm{a}$ & $0,05 \mathrm{a}$ & $0,38 \mathrm{a}$ \\
PFA & $1,77 \mathrm{~b}$ & $1,65 \mathrm{~b}$ & $1,35 \mathrm{~b}$ & $1,02 \mathrm{a}$ & $0,03 \mathrm{a}$ & $0,44 \mathrm{a}$ \\
PFV & $1,42 \mathrm{~b}$ & $1,66 \mathrm{~b}$ & $2,06 \mathrm{a}$ & $1,00 \mathrm{a}$ & $0,02 \mathrm{a}$ & $0,46 \mathrm{a}$ \\
\hline C.V. & \multicolumn{7}{c}{13,77} \\
\hline D.M.S. & \multicolumn{7}{c}{0,31} \\
\hline F & \multicolumn{7}{c}{5,60} \\
\hline
\end{tabular}

Em que: Médias seguidas de letras iguais não diferem significativamente entre si pelo teste de Tukey a $0,05 \%$ de probabilidade. Letras minúsculas referem-se à comparação vertical (tratamento dentro de época de avaliação); Em que: APF: ausência de protetor físico; PFT: protetor físico transparente; PFA: protetor físico azul; PFV: protetor físico vermelho.

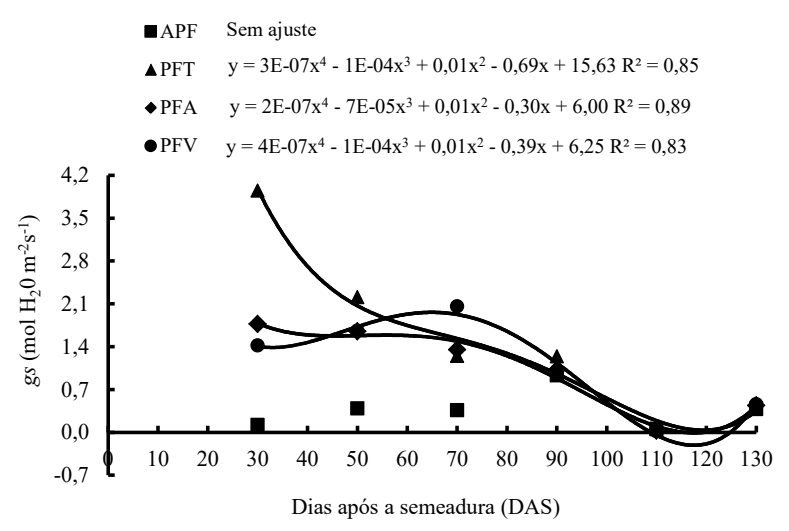

FIGURA 1: Valores médios da condutância estomática $\left(g_{s}, \mathrm{mmolH}_{2} \mathrm{O} \mathrm{m}^{-2} \mathrm{~s}^{-1}\right)$, de folhas de plantas de canafístula [Peltophorum dubium (Spreng.) Taub.], submetidas a diferentes qualidades de luminosidade: Em que: APF: ausência de protetor físico; PFT: protetor físico transparente; PFA: protetor físico azul; PFV: protetor físico vermelho.

FIGURE 1: Mean values of stomatal conductance $\left(g_{s}, \mathrm{mmol} \mathrm{H}_{2} \mathrm{O} \mathrm{m}^{-2} \mathrm{~s}^{-1}\right)$, leaves of seedlings of canafistula [Peltophorum dubium (Spreng.) Taub.], subjected to different qualities of light: Where: APF: no physical guard; PFT: physical guard transparent; PFA: physical shield blue; PFV: red guard physical. rapidamente, com a finalidade de restringir a perda de água. Tal processo foi detectado no presente experimento mesmo com o solo úmido. Sob condições ambientais mais favoráveis, as trocas gasosas determinadas aos 130 DAS não apresentaram variação entre os tratamentos (Tabela 1 e Figura 1).

De forma geral, a retirada do protetor físico promoveu menor variação no comportamento da condutância estomática. Visualmente, notou-se que a retirada dos protetores levou à desidratação das folhas, principalmente daquelas mais externas. Porém, a mortalidade de algumas dessas folhas estimulou novas brotações, o que foi mais visível nos tratamentos com protetores físicos azuis e vermelhos. Tal resposta, provavelmente ocorreu devido à estratégia utilizada pelas plantas em relação à redução de luminosidade, as quais preferiram prioritariamente na expansão do limbo foliar e na espessura das folhas, interferindo na perda de água. Segundo Paiva et al. (2005), o controle da abertura e fechamento estomático é uma importante propriedade fisiológica, pelo qual as plantas podem limitar sua perda de água.

$\mathrm{Da}$ mesma forma, Costa e Marenco (2007) observaram que a máxima assimilação em Carapa guianensis ocorreu no início da manhã, demonstrando que os fatores ambientais podem afetar o potencial hídrico refletindo diretamente nas 
trocas gasosas.

\section{Transpiração}

Os valores médios da taxa de transpiração das folhas foram maiores na presença dos protetores físicos, independentemente de sua coloração, entre 30 e 70 DAS (Tabela 2 e Figura 2). Tal comportamento era esperado, visto que a condutância estomática em plantas mantidas sem proteção (APF) foi significativamente menor que a das demais plantas durante este período. $\mathrm{O}$ aumento da transpiração observado nas plantas mantidas com protetores físicos foi devido às condições mais favoráveis de temperatura e umidade no interior dos protetores.

Plantas de C. vernalis foram cultivadas sob níveis elevados de sombreamento e apresentaram maior área foliar, que levou à maior transpiração destas plantas em comparação àquelas cultivadas a pleno sol (LIMA-JÚNIOR et al., 2006).

Tais resultados são diferentes daqueles obtidos por Kjelgren (1994) que observou valores menores de transpiração de plantas de café cultivadas no interior de protetores físicos de plástico. Segundo o mesmo autor, elevados valores de temperatura e umidades no interior dos protetores físicos promovem redução na transpiração.

No período de 50 DAS, o protetor físico transparente levou aos maiores valores desta característica, seguido pelos tratamentos com protetores físicos vermelho e azul. Durante o período em que havia protetores físicos, até os 70 DAS, os maiores valores obtidos para a transpiração foram observados aos 50 DAS em todas as plantas.

No entanto, aos 90 DAS, vinte dias após a retirada dos protetores físicos, foram detectadas menores taxas de transpiração nas plantas que haviam sido mantidas em tais protetores. Porém, devido à desidratação visualmente observada nas folhas das plantas crescidas no interior dos protetores nos períodos de 90 e 110 DAS, não é possível afirmar que somente as condições ambientais internas de tais protetores tenham sido responsáveis pelos maiores valores médios de transpiração observados nas avaliações anteriores nestes tratamentos. Tal fato pode ser constatado através da sensibilidade das folhas das plantas após a retirada dos protetores, evidenciada pela redução da transpiração.

Nesse sentido, torna-se relevante mencionar que a condutância estomática observada entre os $90 \mathrm{e}$ 130 DAS não apresentou diferença significativa entre os tratamentos (Tabela 1 e Figura 1), enquanto que a transpiração das folhas na presença de protetores físicos, independentemente de sua coloração, foi significativamente menor durante o mesmo período (Tabela 2 e Figura 2). Provavelmente, as folhas das plantas desenvolvidas no interior dos protetores físicos foram menos tolerantes às elevadas taxas de luminosidade, respondendo rapidamente por meio do fechamento estomático.

Em relação aos modelos matemáticos

TABELA 2: Valores médios da transpiração foliar $\left(E\right.$, mmol $\left.\mathrm{H}_{2} \mathrm{O} \mathrm{m} \mathrm{m}^{-2} \mathrm{~s}^{-1}\right)$ em plantas de canafístula [Peltophorum dubium (Spreng.) Taub.], submetidas a diferentes qualidades de luminosidades.

TABLE 2: Mean values of leaf transpiration $\left(E, \mathrm{mmol} \mathrm{H}_{2} \mathrm{O} \mathrm{m}^{-2} \mathrm{~s}^{-1}\right)$, leaves of seedlings of canafístula [Peltophorum dubium (Spreng.) Taub.], subjected to different qualities of light.

\begin{tabular}{ccccccc}
\hline \multirow{2}{*}{ Tratamento } & \multicolumn{7}{c}{ Dias após a semeadura (DAS) } \\
\cline { 2 - 7 } & 30 & 50 & 70 & 90 & 110 & 130 \\
\hline APF & $1,22 \mathrm{~b}$ & $8,48 \mathrm{c}$ & $6,21 \mathrm{~b}$ & $6,64 \mathrm{a}$ & $2,27 \mathrm{a}$ & $8,48 \mathrm{a}$ \\
PFT & $5,13 \mathrm{a}$ & $13,59 \mathrm{a}$ & $9,18 \mathrm{a}$ & $3,20 \mathrm{c}$ & $1,87 \mathrm{ab}$ & $4,48 \mathrm{~b}$ \\
PFA & $4,69 \mathrm{a}$ & $11,70 \mathrm{~b}$ & $8,99 \mathrm{a}$ & $3,84 \mathrm{c}$ & $1,47 \mathrm{ab}$ & $3,86 \mathrm{~b}$ \\
PFV & $4,26 \mathrm{a}$ & $11,76 \mathrm{~b}$ & $9,61 \mathrm{a}$ & $4,56 \mathrm{~b}$ & $0,98 \mathrm{~b}$ & $3,88 \mathrm{~b}$ \\
\hline C.V. & \multicolumn{7}{c}{0,36} \\
\hline D.M.S. & 0,69 \\
\hline F & $27,85^{* *}$ \\
\hline
\end{tabular}

Em que: Médias seguidas de letras iguais não diferem significativamente entre si pelo teste de Tukey a $0,01 \%$ de probabilidade. Letras minúsculas referem-se à comparação vertical (tratamento dentro de época de avaliação); Em que: APF: ausência de protetor físico; PFT: protetor físico transparente; PFA: protetor físico azul; PFV: protetor físico vermelho. 


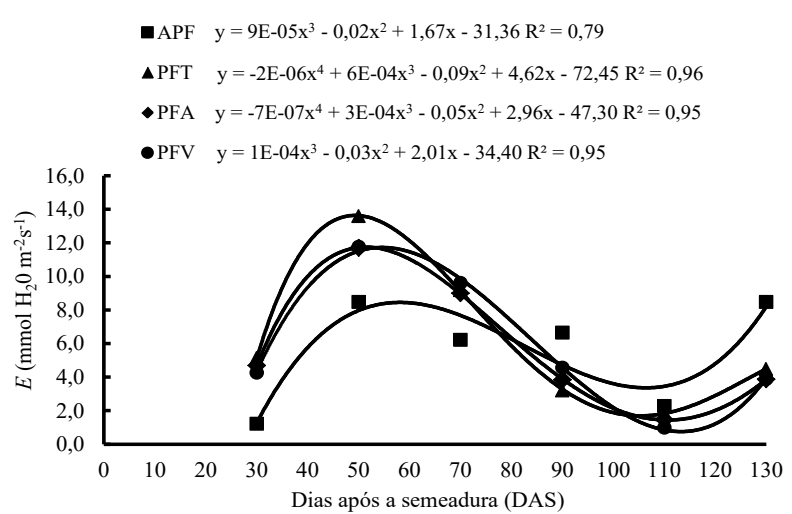

FIGURA 2: Valores médios da transpiração foliar $\left(E\right.$, mmol $\left.\mathrm{H}_{2} \mathrm{O} \mathrm{m}^{-2} \mathrm{~s}^{-1}\right)$ de plantas de [Peltophorum dubium (Spreng.) Taub.], submetidas a diferentes qualidades de luminosidade: Em que: APF: ausência de protetor físico; PFT: protetor físico transparente; PFA: protetor físico azul; PFV: protetor físico vermelho.

FIGURE 2: Mean values of leaf transpiration $(E$,

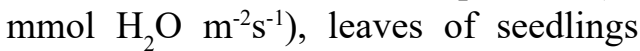
of canafístula [Peltophorum dubium (Spreng.) Taub.], subjected to different qualities of light: Where: APF: no physical guard; PFT: physical guard transparent; PFA: physical shield blue; PFV: red guard physical.

ajustados para a transpiração em função dos seis períodos de avaliações, adotaram-se o modelo de regressão polinomial de terceiro grau aos tratamentos APF e PFV, enquanto que para os tratamentos PFT e PFA, utilizaram-se modelos de quarto grau.

\section{Taxa de assimilação líquida de $\mathrm{CO}_{2}$}

Foi detectada interação significativa entre protetores físicos e dias após a semeadura, a $1 \%$ de probabilidade para a assimilação líquida de $\mathrm{CO}_{2}$ (Tabela 3 e Figura 3). Somente aos 30 DAS, o tratamento com ausência de protetor físico atingiu os menores valores da taxa de assimilação líquida de $\mathrm{CO}_{2}$, provavelmente devido à redução no tamanho das plantas de canafístula crescidas na ausência dos protetores físicos. As plantas submetidas ao protetor físico vermelho, apesar de apresentarem maior área foliar, tiveram os menores valores médios da taxa de assimilação líquida de $\mathrm{CO}_{2}$ a partir dos 50 DAS.

No período de avaliação da taxa de assimilação líquida de $\mathrm{CO}_{2}$, após a retirada dos protetores físicos, entre 90 e 130 DAS, também foram observadas diferenças significativas desta característica. Os primeiros 20 dias após a retirada dos protetores físicos (90 DAS) não foram suficientes para influenciar na taxa de assimilação líquida de $\mathrm{CO}_{2}$ das plantas provenientes dos tratamentos com protetores físicos transparente e azul, pois as mesmas apresentaram valores semelhantes àqueles detectados nas plantas provenientes sem proteção (APF).

Estes resultados não eram esperados, pois as plantas de canafístula cultivadas no interior dos protetores físicos, principalmente transparente e azul, apresentaram valores elevados de taxa de assimilação líquida de $\mathrm{CO}_{2}$ mesmo após a redução da taxa de transpiração (Figura 2) e condutância estomática (Figura 1). Estes resultados são semelhantes àqueles observados por Habermann et al. $(2003,2008)$ ao avaliarem o comportamento das trocas gasosas de laranjeira-doce e limeira-ácida (SANTOS et al., 2011), visto que o aumento da resistência estomática foi mais eficiente no controle da transpiração do que na assimilação líquida de $\mathrm{CO}_{2}$.

Da mesma forma, pode-se observar no presente estudo que a transpiração foi mais influenciada pelo fechamento estomático (Figura 1) do que pela assimilação líquida de $\mathrm{CO}_{2}$ (Figura 3 ).

A redução na quantidade de luminosidade e de temperatura aos 90 e 110 DAS devido à presença de nuvens e de vento auxiliaram na redução desta característica durante o referido período, o que não justifica os valores reduzidos da taxa de assimilação líquida de $\mathrm{CO}_{2}$ observados nas plantas aos 110 DAS. Tais valores podem estar correlacionados à resposta das folhas em função do aumento da luminosidade após a retirada dos protetores físicos. Além disso, a mortalidade de alguns folíolos foi observada durante este período em todas as plantas mantidas no interior de protetores físicos após a remoção do protetor físico. Neste sentido, o trabalho realizado por Dias e Marenco (2007) com mogno (Swietenia macrophylla King) e acariquara (Minquartia guianensis Aubl.) demonstrou que uma hora de exposição à alta temperatura $\left(38^{\circ} \mathrm{C}\right)$ e elevada radiação causa diminuição na taxa de assimilação liquida de $\mathrm{CO}_{2}$ em razão da redução da condutância estomática.

Aos 130 DAS, as plantas de canafístula crescidas sob os protetores físicos apresentaram valores mais elevados em relação àqueles observados 
aos 110 DAS, porém, inferiores aos obtidos nas folhas das plantas do tratamento testemunha (APF), apresentando melhoras visuais na qualidade das folhas com brotações novas.

Independentemente da coloração do protetor físico utilizado, o modelo de regressão polinomial mais adequado em relação à assimilação líquida de $\mathrm{CO}_{2}$ ao longo das diferentes épocas de avaliação foi o de quarto grau, enquanto que para a ausência de protetor físico o melhor ajuste foi o modelo de terceiro grau (Figura 3).

Diferentemente, em trabalho realizado com cascudo (Talisia sulbalbens (Mart.) Radlk.), plantas cultivadas sob diferentes níveis de luminosidade observaram que mudas cultivadas a pleno sol e a $30 \%$ de sombreamento apresentaram maior desenvolvimento de plantas, sem interferir nos valores de condutância estomática e fotossíntese entre os níveis de sombreamento nos períodos de avaliação (NERY et al., 2011).

\section{Concentração de $\mathrm{CO}_{2}$ dentro da câmara subestomática $(\mathrm{C} i)$}

A concentração de $\mathrm{CO}_{2}$ na câmara subestomática $(\mathrm{Ci})$ de folhas de canafístula foi alterada com a presença do protetores físicos, independentemente da coloração utilizada. Assim, maiores valores de $\mathrm{Ci}$ foram detectadas nas plantas mantidas sob protetores físicos até os 90 DAS (Tabela 4 e Figura 4).

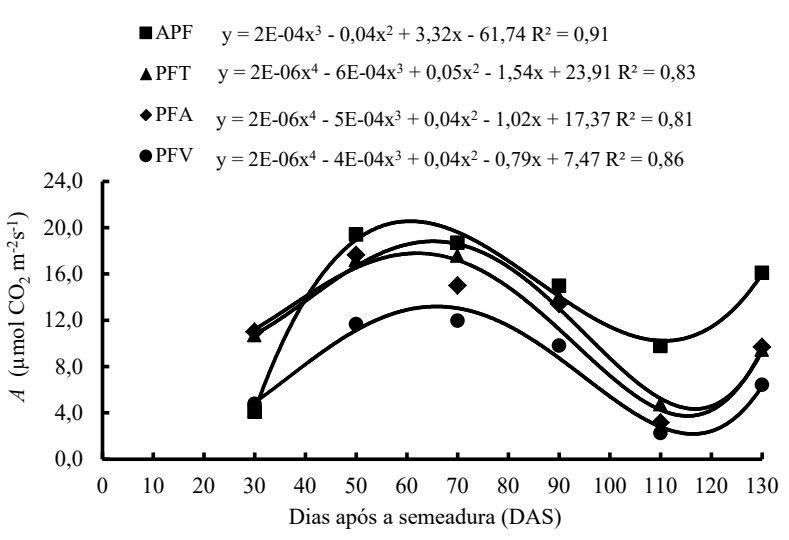

FIGURA 3: Valores médios da taxa de assimilação de $\mathrm{CO}_{2}\left(A, \mu \mathrm{mol} \mathrm{CO} \mathrm{m}^{-2} \mathrm{~s}^{-1}\right)$ de plantas de [Peltophorum dubium (Spreng.) Taub.], submetidas a diferentes qualidades de luminosidade: Em que: APF: ausência de protetor físico; PFT: protetor físico transparente; PFA: protetor físico azul; PFV: protetor físico vermelho.

FIGURE 3: Mean values of net assimilation rate of $\mathrm{CO}_{2}\left(A, \mu \mathrm{mol} \mathrm{CO} \mathrm{m}^{-2} \mathrm{~s}^{-1}\right)$, leaves of seedlings of canafístula [Peltophorum dubium (Spreng.) Taub.], subjected to different qualities of light: Where: APF: no physical guard; PFT: physical guard transparent; PFA: physical shield blue; PFV: red guard physical.

TABELA 3: Valores médios da taxa de assimilação líquida de $\mathrm{CO}_{2}\left(A, \mu \mathrm{mol} \mathrm{CO}_{2} \mathrm{~m}^{-2} \mathrm{~s}^{-1}\right)$ em plantas de canafístula [Peltophorum dubium (Spreng.) Taub.], submetidas a diferentes qualidades de luminosidade.

TABLE 3: Mean values of net assimilation rate of $\mathrm{CO}_{2}\left(A, \mu \mathrm{mol} \mathrm{CO}_{2} \mathrm{~m}^{-2} \mathrm{~s}^{-1}\right)$, leaves of seedlings of canafístula [Peltophorum dubium (Spreng.) Taub.], subjected to different qualities of light.

\begin{tabular}{ccccccc}
\hline \multirow{2}{*}{ Tratamento } & \multicolumn{7}{c}{ Dias após a semeadura } \\
\cline { 2 - 7 } & 30 & 50 & 70 & 90 & 110 & 130 \\
\hline APF & $4,09 \mathrm{~b}$ & $19,40 \mathrm{a}$ & $18,71 \mathrm{a}$ & $14,99 \mathrm{a}$ & $9,77 \mathrm{a}$ & $16,10 \mathrm{a}$ \\
PFT & $10,69 \mathrm{a}$ & $17,18 \mathrm{a}$ & $17,57 \mathrm{ab}$ & $14,03 \mathrm{a}$ & $4,73 \mathrm{~b}$ & $9,45 \mathrm{~b}$ \\
PFA & $11,00 \mathrm{a}$ & $17,64 \mathrm{a}$ & $15,00 \mathrm{~b}$ & $13,42 \mathrm{a}$ & $3,16 \mathrm{~b}$ & $9,70 \mathrm{~b}$ \\
PFV & $4,79 \mathrm{~b}$ & $11,68 \mathrm{~b}$ & $11,96 \mathrm{c}$ & $9,81 \mathrm{~b}$ & $2,25 \mathrm{~b}$ & $6,42 \mathrm{c}$ \\
\hline C.V. & \multicolumn{7}{c}{15,32} \\
\hline D.M.S. & 0,61 & & \\
\hline F & 8,10 & \\
\hline
\end{tabular}

Em que: Médias seguidas de letras iguais não diferem significativamente entre si pelo teste de Tukey a $0,01 \%$ de probabilidade. Letras minúsculas referem-se à comparação vertical (tratamento dentro de época de avaliação); Em que: APF: ausência de protetor físico; PFT: protetor físico transparente; PFA: protetor físico azul; PFV: protetor físico vermelho. 
Diversas hipóteses disponíveis na literatura podem ser usadas para explicar o comportamento da $C i$ observado no presente estudo. A primeira refere-se ao aumento da $\mathrm{Ci}$ em função da redução da resistência estomática (Figura 4) promovida pelo aumento da condutância estomática (Figura 1) (TAIZ e ZEIGER, 2013). A resistência estomática, conforme os mesmos autores, é o inverso da condutância estomática, ou seja, são processos físicos. Assim, quanto maior a abertura dos estômatos, maior a probabilidade de entrada de $\mathrm{CO}_{2}$ para o interior da folha. A segunda possibilidade é atribuída às altas taxas de concentração de $C i$ dentro das câmaras subestomáticas pela redução do consumo de $\mathrm{CO}_{2}$ pelas células vizinhas, assim como pelas células do parênquima, ou seja, de maneira metabólica. Este processo pode estar diretamente relacionado à quantidade de carbono fixado pelos cloroplastos no final da fotossíntese, ou seja, quanto menor a taxa assimilação (Figura 3), maior a concentração de $\mathrm{CO}_{2}$ (Figura 4) no interior da câmara subestomática.

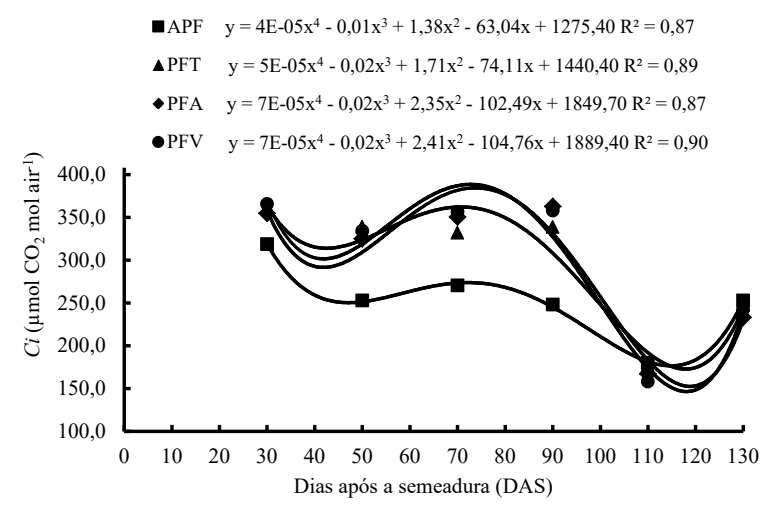

FIGURA 4: Valores médios da concentração interna de $\mathrm{CO}_{2}(\mathrm{Ci}, \mu \mathrm{mol}$ mol air-1), da câmara subestomática de plantas de canafístula [Peltophorum dubium (Spreng.) Taub.], submetidas a diferentes qualidades de luminosidade: Em que: APF: ausência de protetor físico; PFT: protetor físico transparente; PFA: protetor físico azul; PFV: protetor físico vermelho.

FIGURE 4: Mean values of $\mathrm{CO}_{2}$ concentration $(\mathrm{Ci}$, $\mu \mathrm{mol}$ mol air-1), within the substomatal chamber seedlings of canafístula [Peltophorum dubium (Spreng.) Taub.], subjected to different qualities of light: Where: APF: no physical guard; PFT: physical guard transparent PFA: physical shield blue; PFV: red guard physical.
No entanto, Galon et al. (2010) consideram a $C i$ como uma característica influenciada por fatores ambientais como a disponibilidade hídrica, luz, temperatura e energia, entre outros.

A presença de protetor físico independentemente de sua coloração proporcionou incremento nos valores médios de $C i$ durante o período de 30 e 90 DAS. No entanto, estas diferenças foram reduzidas ao final de 110 dias, pois não foi possível verificar diferenças significativas para esta variável até o final do experimento 130 DAS. Estes resultados são importantes, principalmente quando comparados àqueles obtidos pela assimilação de dióxido de carbono (Tabela 3, Figura 3). Nota-se que a variável $C i$ se mostrou mais sensível perante as imposições aplicadas comparadas aos valores médios de assimilação líquida de $\mathrm{CO}_{2}$.

Nas avaliações realizadas aos 110 e 130 DAS, não houve influência do protetor físico na concentração interna de $\mathrm{CO}_{2}$ na câmara subestomática (Tabela 4 e Figura 4). Em todos os tratamentos, o modelo de regressão polinomial ajustado foi o de quarto grau.

\section{Eficiência do uso de água $(E U A)$}

Na Tabela 5 e na Figura 5 encontramse os resultados referentes à eficiência do uso de água $(E U A)$ de plantas de canafístula. A EUA das folhas foi afetada pela presença do protetor físico, independentemente de sua coloração. Quanto à interação entre os protetores físicos e as épocas de avaliação, verificou-se que a mesma foi significativa a $5 \%$ de probabilidade. Aos 30 e 90 DAS foram detectados os maiores valores médios de $E U A$ em todas as plantas.

No período em que o protetor físico estava presente no ponto de semeadura, até os 70 DAS, foi observada a influência deste material, independentemente de sua coloração sobre a $E U A$ das plantas de canafístula. Assim, os protetores físicos empregados proporcionaram redução da $E U A$ das plantas crescidas no seu interior. Tal comportamento deve-se aos altos valores da taxa de transpiração (Figura 2) observados nas plantas submetidas à presença de protetores. Estes resultados corroboram os de Larcher (2000) que afirma que a $E U A$ pode sofrer mudanças quando a difusão de $\mathrm{CO}_{2}$ e $\mathrm{H}_{2} \mathrm{O}$ é alterada por mudanças nas condições ambientais. O mesmo autor afirma ainda que a EUA expressa quantitativamente o comportamento momentâneo das trocas gasosas na folha. Além disso, a EUA pode 
TABELA 4: Valores médios da concentração de $\mathrm{CO}_{2}(\mathrm{Ci}, \mu \mathrm{mol}$ mol air-1), no interior da câmara subestomática de plantas de canafístula [Peltophorum dubium (Spreng.) Taub.], submetidas a diferentes qualidades de luminosidade.

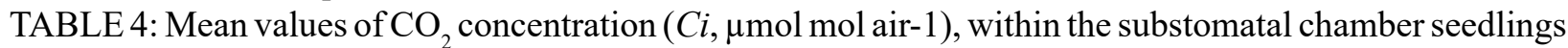
of canafístula [Peltophorum dubium (Spreng.) Taub.], subjected to different qualities of light.

\begin{tabular}{ccccccc}
\hline \multirow{2}{*}{ Tratamento } & \multicolumn{7}{c}{ Dias após a semeadura } \\
\cline { 2 - 7 } & 30 & 50 & 70 & 90 & 110 & 130 \\
\hline APF & $318,60 \mathrm{~b}$ & $252,80 \mathrm{~b}$ & $270,40 \mathrm{~b}$ & $248,00 \mathrm{~b}$ & $180,00 \mathrm{a}$ & $252,80 \mathrm{a}$ \\
PFT & $360,80 \mathrm{a}$ & $338,80 \mathrm{a}$ & $331,60 \mathrm{a}$ & $338,60 \mathrm{a}$ & $176,60 \mathrm{a}$ & $246,80 \mathrm{a}$ \\
PFA & $354,60 \mathrm{a}$ & $325,00 \mathrm{a}$ & $350,20 \mathrm{a}$ & $362,60 \mathrm{a}$ & $167,20 \mathrm{a}$ & $233,00 \mathrm{a}$ \\
PFV & $365,60 \mathrm{a}$ & $334,20 \mathrm{a}$ & $356,40 \mathrm{a}$ & $357,80 \mathrm{a}$ & $158,20 \mathrm{a}$ & $242,20 \mathrm{a}$ \\
\hline C.V. & \multicolumn{7}{c}{14,97} \\
\hline D.M.S. & 1,60 \\
\hline F & 9,35 & & \\
\hline
\end{tabular}

Em que: Médias seguidas de letras iguais não diferem significativamente entre si pelo teste de Tukey a $0,01 \%$ de probabilidade. Letras minúsculas referem-se à comparação vertical (tratamento dentro de época de avaliação); Em que: APF: ausência de protetor físico; PFT: protetor físico transparente; PFA: protetor físico azul; PFV: protetor físico vermelho.

TABELA 5: Valores médios da eficiência do uso da água $\left(E U A, \mu \mathrm{mol} \mathrm{CO}_{2} \mathrm{~m}^{-2} \mathrm{~s}^{-1}\left(\mathrm{mmol} \mathrm{H}_{2} \mathrm{O} \mathrm{m}^{-2} \mathrm{~s}^{-1}\right)^{-1}\right)$ de plantas de canafístula [Peltophorum dubium (Spreng.) Taub.], submetidas a diferentes qualidades de luminosidade.

TABLE 5: Mean values of the efficiency of water use $\left(E U A, \mu \mathrm{mol} \mathrm{CO}_{2} \mathrm{~m}^{-2} \mathrm{~s}^{-1}\left(\mathrm{mmol} \mathrm{H}_{2} \mathrm{O} \mathrm{m}^{-2} \mathrm{~s}^{-1}\right)^{-1}\right)$, leaves of seedlings of canafístula [Peltophorum dubium (Spreng.) Taub.], subjected to different qualities of light.

\begin{tabular}{ccccccc}
\hline \multirow{2}{*}{ Tratamento } & \multicolumn{7}{c}{ Dias após a semeadura (DAS) } \\
\cline { 2 - 7 } & 30 & 50 & 70 & 90 & 110 & 130 \\
\hline APF & $3,32 \mathrm{a}$ & $2,31 \mathrm{a}$ & $3,06 \mathrm{a}$ & $2,29 \mathrm{c}$ & $4,70 \mathrm{a}$ & $1,91 \mathrm{a}$ \\
PFT & $2,10 \mathrm{~b}$ & $1,27 \mathrm{~b}$ & $1,91 \mathrm{~b}$ & $4,47 \mathrm{a}$ & $2,56 \mathrm{~b}$ & $2,11 \mathrm{a}$ \\
PFA & $2,36 \mathrm{~b}$ & $1,52 \mathrm{~b}$ & $1,68 \mathrm{~b}$ & $3,55 \mathrm{~b}$ & $2,19 \mathrm{~b}$ & $2,53 \mathrm{a}$ \\
PFV & $1,12 \mathrm{c}$ & $0,99 \mathrm{bc}$ & $1,27 \mathrm{~b}$ & $2,17 \mathrm{c}$ & $2,37 \mathrm{~b}$ & $1,65 \mathrm{a}$ \\
\hline C.V. & \multicolumn{7}{c}{9,56} \\
\hline D.M.S. & \multicolumn{7}{c}{0,51} \\
\hline F & \multicolumn{7}{c}{8,87} \\
\hline
\end{tabular}

Em que: Médias seguidas de letras iguais não diferem significativamente entre si pelo teste de Tukey a $0,05 \%$ de probabilidade. Letras minúsculas referem-se à comparação vertical (tratamento dentro de época de avaliação); Em que: APF: ausência de protetor físico; PFT: protetor físico transparente; PFA: protetor físico azul; PFV: protetor físico vermelho.

interferir na produtividade biológica bem como no consumo hídrico (PIMENTEL, 2004).

Há evidência de que a EUA pode variar entre espécies situadas no mesmo ambiente e entre diferentes condições climáticas (SILVA et al., 2004). Na literatura, diversos autores têm avaliado a
EUA para melhor conhecimento do comportamento de espécies cultivadas a fim de aumentar sua produtividade, principalmente naquelas submetidas aos diferentes níveis hídricos (STONE e MOREIRA, 2000; MEDEIROS et al., 2001; KARAM et al., 2003; TENNAKOON e MILROY, 2003). Além 


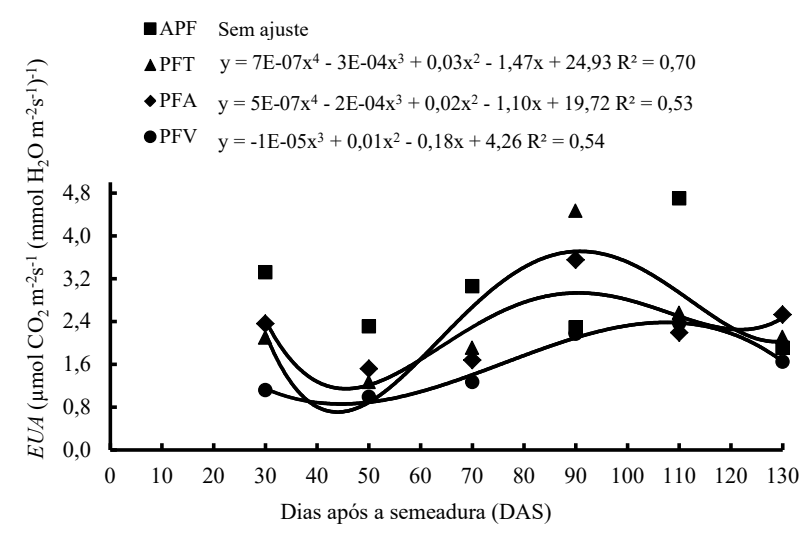

FIGURA 5: Valores médios da eficiência do uso de água $\left(E U A, \mu \mathrm{mol} \mathrm{CO}_{2} \mathrm{~m}^{-2} \mathrm{~s}^{-1} / \mathrm{mmol}\right.$ $\left.\mathrm{H}_{2} \mathrm{O} \mathrm{m}^{-2} \mathrm{~s}^{-1}\right)$ da câmara subestomática de plantas de canafístula [Peltophorum dubium (Spreng.) Taub.], submetidas a diferentes qualidades de luminosidade: Em que: APF: ausência de protetor físico; PFT: protetor físico transparente; PFA: protetor físico azul; PFV: protetor físico vermelho.

FIGURE 5: Mean values of the efficiency of water use $\left(E U A, \mu \mathrm{mol} \mathrm{CO} \mathrm{C}_{2}^{-2} \mathrm{~s}^{-1}\left(\mathrm{mmol} \mathrm{H}_{2} \mathrm{O}\right.\right.$ $\left.\left.\mathrm{m}^{-2} \mathrm{~s}^{-1}\right)^{-1}\right)$, leaves of seedlings of canafístula [Peltophorum dubium (Spreng.) Taub.], subjected to different qualities of light: Where: APF: no physical guard; PFT: physical guard transparent; PFA: physical shield blue; PFV: red guard physical.

disso, busca-se por meio desta característica incluir as espécies arbóreas, que são mais resistentes à redução da disponibilidade de água, em projetos de reflorestamento tanto para finalidades econômicas quanto comerciais (SILVA et al., 2004). Porém, são raros os estudos que quantificam a $E U A$ em função dos diferentes níveis de luminosidade.

De forma geral, o protetor físico transparente utilizado promoveu maior incremento nos valores das trocas gasosas das folhas das plantas de canafístula (Peltophorum dubium), com máxima abertura estomática durante o período de permanência do protetor (70 dias após a semeadura) (Figura 1). Esta condição possibilitou maior valor da taxa de transpiração, como pode ser observado na Figura 2. Por consequência, os valores médios da taxa de assimilação líquida de dióxido de carbono seguiram o mesmo comportamento (Figura $3)$. Independentemente do tipo do protetor físico utilizado, não foi verificado comprometimento do aparato fotossintético, como demonstram os valores médios da concentração interna de dióxido de carbono dentro da câmara subestomática, mesmo com retirada dos protetores físicos (Figura 4). Apenas foi observado redução nos valores de eficiência do uso de água (EUA) nos tratamentos que receberam os protetores físicos (Figura 5); este comportamento é reflexo das boas condições fotossintéticas das folhas, devido à elevada disponibilidade de água no solo. A retirada do protetor físico aos 70 dias após a semeadura (DAS) mostrou-se adequado para as plantas de canafístula.

\section{CONCLUSÕES}

Perante as avaliações de trocas gasosas no presente estudo, a implantação de canafístula (Peltophorum dubium) com o uso de protetores físicos independentemente de sua coloração, proporciona microambiente favorável para o desenvolvimento das plantas até setenta dias após a semeadura, sendo desnecessário seu emprego a partir desse período.

\section{REFERÊNCIAS BIBLIOGRÁFICAS}

AGRITEMPO. Sistema de monitoramento agrometeorológico. Disponível em: $<\mathrm{http}: / / \mathrm{www}$. agritempo.gov.br/>. Acesso em: 30 jul. 2011.

AVILA, A. L. et al. Caracterização da vegetação e espécies para recuperação de mata ciliar. Ciência Florestal, Santa Maria, v.21, n.2, p.251-260, 2011. BANZATTO, D.A.; KRONKA, S.N. Experimentação agrícola. Jaboticabal, FUNEP, 1989, 247 p.

BRACHTVOGEL, E.L.; KLEIN, J.; MALAVASI, U.C. Semeadura direta de espécies florestais com protetores físicos - Uma revisão. Ambiência, Guarapuava, v.4, n.3, p.519-527, 2008.

BARNETT, J.P.; BAKER, J.B. Regeneration Methods. In: DURYEA, M.L.; DOUGHERTY, P.M. (Ed) Forest regeneration manual. Kluwer Academic Publishers, London, v. 3, p.35-50, 1991.

COSTA, G.F.; MARENCO, R.A. Fotossíntese, condutância estomática e potencial hídrico foliar em árvores jovens de andiroba (Carapa guianensis). Acta Amazonica, Manaus, v.37, n.2, p.229-234, 2007.

DERR, H.J.; MANN JR., W.F. Direct seeding pines in the south. Washington, DC : USDA. Forest 
service, 1971. 68p. (Agricultural Handbook, 391). DIAS, D.P.; MARENCO, R.A. Fotossíntese e fotoinibição em mogno e acariquara em função da luminosidade e temperatura foliar. Pesquisa Agropecuária Brasileira, Brasília, v.42, n.3, p.305-311, 2007.

DONADIO, N.M.M.; DEMATTÊ, M.E.S.P. Morfologia de frutos, sementes, e plântulas de canafístula (Peltophorum dubium (Spreng.) Taub.) e jacarandá-da-Bahia (Dalbergia nigra (Vell.) Fr. All. ex Benth.) - Fabaceae. Revista Brasileira de Sementes, Brasília, v.22, n.1, p.64-73, 2000.

DOUSSEAU, $S$. et al. Influência de diferentes condições de sombreamento sobre o crescimento de Tapirira guianensis Alb. Revista Brasileira de Biociências, Porto Alegre, v.5, s.1, p.447-479, 2007. EMBRAPA - EMPRESA BRASILEIRA DE PESQUISA AGROPECUÁRIA. Sistema Brasileiro de Classificação de Solos. 3.ed. Brasília, Embrapa, 2013. 353p.

FERRAZ-GRANDE, F.G.A.; TAKAKI, M. Efeitos da luz, temperatura e estresse de água na germinação de sementes de Caesalpinia peltophoroides Benth. (Caesalpinoideae). Bragantia, Campinas, v.65, n.1 , p.37-42, 2006.

FERREIRA, R.A.; DAVIDE, A.C.; MOTTA, M. S. Vigor e viabilidade de sementes de Senna multijuga (Rich.) et Barn., num banco de sementes em solo de viveiro. Revista Brasileira de Sementes, v. 26, n.1, p.24-31, 2004

FERREIRA, D.F. SISVAR: A computer statistical analysis system. Ciência e Agrotecnologia, Lavras, v.15, n.6, p.1039-1042, 2011.

FERREIRA, R.A. et al. Semeadura direta com espécies arbóreas para recuperação de ecossistemas florestais. Cerne, Lavras, v.13, n.3, p.271-279, 2007. FREITAS, R.B. Influência de diferentes níveis de sombreamento no comportamento fisiológico de cultivares de café (Coffea arabica). Ciência e Agrotecnologia, Lavras, v.27, n.4, p.804-810, 2003. GALON, L. et al. Eficiência de uso da água em genótipos de cana-de-açúcar submetidos à aplicação de herbicidas. Planta daninha, Viçosa, v.28, n.4, p.777-784, 2010.

HABERMANN, G. et al. $\mathrm{CO}_{2}$ assimilation, photosynthetic light response curves, and water relations of Pêra sweet orange plants infected with Xylella fastidiosa. Brazilian Journal of Plant Physiology. Londrina, v.31, n.2, p.79-87, 2003.

HABERMANN, G. et al. Leaf heliotropism in Styrax camporum Pohl from the Brazilian cerrado distinct gas exchange and leaf structure, but similar leaf temperature and water relations. Brazilian Journal of Plant Physiology, Londrina, v.20, n.1, p.71-83, 2008.

HANSEN, M.C. et al. Humid tropical forest clearing from 2000 to 2005 quantified by using multitemporal and multiresolution remotely sensed data. PNAS, 105: 9439-9444, 2008.

INPE. Instituto Nacional de Pesquisas Espaciais. Desmatamento em São Paulo aumentou nove vezes desde 2005. http://chicaodoispassos.blogspot. com/2008/12/inpe-desmatamento-em-so-pauloaumentou.html, 2009.

KARAM, F. et al.. Evapotranspiration, yield and water use efficiency of drip irrigated corn in the Bekaa Valley of Lebanon. Agricultural and Water Management, Amsterdam, v. 63, n. 2, p.125-137, 2003.

KINOSHITA, T. et al. Phot 1 and phot 2 mediate blue light regulation of stomatal opening. Nature, v.414, n.6864, p.656-660, 2001.

KJELGREN, R. Growth and water relations of Kentucky coffee tree in protective shelters during establishment. HortScience, v.29, n.7, p.777-780, 1994.

KLEIN, J. et al. Influence of light quality on germination and initial growth of canephori seedling [Peltophorum dubium (Sprenge) Taub.]. International Journal of Food, Agriculture and Environment, v.10, p. 947-951, 2012.

LARCHER, W. Ecofisiologia vegetal. São Carlos, RiMa, 337 p, 2004.

LIMA-JUNIOR, É.C. Trocas gasosas, caracterísitcas das folhas e crescimento de plantas jovens de Cupania vernalis Camb. Submetidas a diferentes níveis de sombreamento. Ciência Rural, Santa Maria, v.35, n.5, p.1092-1097, 2005.

LORENZI, H. Árvores brasileiras.3. ed. Nova Odesa-SP, 352 p, 2000.

MALAVASI, U.C., KLEIN, J.; MALAVASI, M.M. Efeito de um protetor físico na semeadura direta de duas espécies florestais em área de domínio ciliar. Revista Árvore, Viçosa, v.34, n.5, p.781-787, 2010. MARTINS, J.R. Avaliação do crescimento e do teor de óleo essencial em plantas de Ocimum gratissimum L. cultivadas sob malhas coloridas. Revista Brasileira de Plantas Medicinais, Botucatu, v.10, n.4, p.102-107, 2008.

MATTEI, V.L. Semeadura direta de Peltophorum dubium (Spreng.) Taub. no enriquecimento de capoeiras. Revista Árvore, Viçosa, v.2, n.1, p.85-96, 1999.

MATTEI, V.L.; ROSENTHAL, M.D. Semeadura 
direta de canafístula (Peltrophorum dubium (Spreng.) taub.) no enriquecimento de capoeiras. Revista Árvore, Viçosa, v.26, n.6, p.649-654, 2002. MATTEI, V.L.; ROMANO, C.M.; TEIXEIRA, M.C.C. Protetores físicos para semeadura direta de Pinus elliotti Engelm. Ciência Rural, Santa Maria, v.31, n.5, p.775-780, 2001.

MEDEIROS, G.A. The influence of crop canopy on evapotranspiration and crop coefficient of beans (Phaseolus vulgaris L.) Agricultural and Water Management, Amsterdam, v.49, n.2, p.215-228, 2001.

NERY, F.C. et al. Initial development and gas exchange of Talisia subalbens (Mart.) Radlk. under different shading conditions. Revista Árvore, Viçosa, v.35, n.1, p.61-67, 2011.

NOGUEIRA, R.J.M.C.; SILVA-JUNIOR, J.F. Resistência estomática, tensão de água no xilema e teor de clorofila em gravioleira (Annona muricata L.). Scientia Agricola, Piracicaba, v.58, n.3, p.491-495, 2001.

NOGUEIRA, R.J.M.C. et al. Comportamiento estomático y tensión de agua en el xilema de dos genotipos de pitanga (Eugenia uniflora L.) cultivados bajo estrés hídrico. Revista de Investigación Agraria Série Producción y Protección Vegetales, Madrid, v.15, n.1, p.213-225, 2000.

PAIVA, A.S. et al. Condutância estomática em folhas de feijoeiro submetido a diferentes regimes de irrigação. Engenharia Agrícola, Jaboticabal, v.25, n.1, p.161-169, 2005.

PIMENTEL, C. A relação da planta com a água.

Seropédica: EDUR, 192 p. 2004.

RAMOS, J.; GRACE, J. The effetcs of shade on the gas exchange of seedlings of four tropical trees from México. Functional Ecology, Oxford, v.4, n.5, p.667-677, 1990.

SANTOS, D.; SIQUEIRA, D.L.; CECON, P.R. Indução floral de limeiras ácidas 'Tahiti' submetidas a baixas temperaturas. Ciencia Rural, Santa Maria, v.41, n.3, p.397-403, 2011.

SERPA, M.R.; MATTEI, V.L. Avaliação de diferentes materiais de cobertura e de um protetor físico, no estabelecimento de plantas de Pinus taeda L., por semeadura direta no campo. Ciência Florestal, Santa Maria, v.9, n.2, p.93-101, 1999.

SILVA, W. et al. Índice de consumo e eficiência do uso da água em eucalipto, submetido a diferentes teores de água em convivência com braquiária. Floresta, Curitiba, 34, n. 3, p.325-335. 2004.

SILVA, R.F. et al. Comportamento de Peltophorum dubium (Spreng.) Taub., Parapiptadenia rigida (Benth.) Brenan e Enterolobium contortisiliquum (Vell.) Morong cultivadas em solo contaminado com cobre. Ciência Florestal, Santa Maria, v.21, n.1, p103-110, 2011.

SOARES, P.G.; RODRIGUES, R.R. Semeadura direta de leguminosas florestais: efeito da inoculação com rizóbio na emergência de plântulas e crescimento inicial no campo. Scientia Forestalis (IPEF), Piracicaba, v.36, n.78, p.115-121, 2008.

STÜRMER, S.L.K. et al. Variações nos teores de carbono orgânico em função do desmatamento e revegetação natural do solo. Ciência Florestal, Santa Maria, v.21, n.2, p.241-250, 2011.

SRIVASTAVA, A.; ZEIGER, E. The inihibitor of zeaxanthin formation, dithiothreitol, inhibits blue-light-stimulated stomatal opening in Vicia faba. Planta, Berlin, v.196, n.2, p.445-449, 1995.

STONE, L.F.; MOREIRA, J.A.A. Efeitos de sistemas de preparo do solo no uso da água e na produtividade do feijoeiro. Pesquisa Agropecuária Brasileira, Brasília, v.35, n.4, p.835-841, 2000.

TAIZ, L.; ZEIGER, E. Fisiologia vegetal. Porto Alegre: Artmed, 2013, 918 p.

TENNAKOON, S.B.; MILROY, S.P. Crop water use efficiency on irrigated cotton farms in Australia. Agricultural and Water Management, Amsterdam, v.61, n.3, p.179-194, 2003. 